\title{
Genetic algorithms based approach for designing spring brake orthosis - Part I: Spring parameters
}

\author{
M.S. Huq ${ }^{\mathrm{a}, *}$ and M.O. Tokhi ${ }^{\mathrm{b}}$ \\ ${ }^{a}$ Department of Mechanical and Aerospace Engineering, Carleton University, Ottawa, ON, Canada \\ ${ }^{\mathrm{b}}$ Department of Automatic Control and Systems Engineering, The University of Sheffield, Sheffield, UK
}

\begin{abstract}
Spring brake orthosis (SBO) concentrates purely on the knee to generate the swing phase of the paraplegic gait with the required hip flexion occurring passively as a consequence of the ipsilateral knee flexion, generated by releasing the torsion spring mounted at the knee joint. Electrical stimulation then drives the knee back to full extension, as well as restores the spring potential energy. In this paper, genetic algorithm (GA) and its variant multi-objective GA (MOGA) is used to perform the search operation for the 'best' spring parameters for the SBO spring mounted on an average sized subject simulated in the sagittal plane. Conventional torsion spring is tested against constant torque type spring in terms of swing duration as, based on first principles, it is hypothesized that constant torque spring would be able to produce slower SBO swing phase as might be preferred in assisted paraplegic gait. In line with the hypothesis, it is found that it is not possible to delay the occurrence of the flexion peak of the SBO swing phase further than its occurrence in the natural gait. The use of conventional torsion spring causes the swing knee flexion peak to appear rather faster than that of the natural gait, resulting in a potentially faster swing phase and hence gait cycle. The constant torque type spring on the other hand is able to stretch duration of the swing phase to some extent, rendering it the preferable spring type in SBO.
\end{abstract}

Keywords: Functional electrical stimulation (FES), genetic algorithm (GA), hybrid orthosis system (HOS), multi-objective genetic algorithm (MOGA), optimization, spring brake orthosis (SBO)

\section{Introduction}

Complete or partial loss of ability to walk or stand due to lower limb paralysis is a very common as well as drastic result of thoracic level spinal cord injury (SCI). The loss of lower limb function and inability to walk or stand significantly reduce the quality of life of disabled individuals and carry with them psychological and physiological effects. The spectrum of problems

*Corresponding author: M.S. Huq, Department of Mechanical and Aerospace Engineering, Carleton University, Ottawa, ON, Canada. E-mail: mshuq@connect.carleton.ca. that may interfere with locomotory performance after an SCI includes hyperactivity of spinal reflex (muscle spasticity) [7], alternation in the muscle activation patterns, including weaknesses and difficulty in coping with weight bearing, balance and gait speed $[35,38]$.

The electrical stimulation of nervous system below the level of the spinal lesion can produce powerful muscle contractions and thus can be used to generate primitive movements. This is termed as functional electrical stimulation (FES) and is used to obtain a functional, useful movement by evoking artificial contraction of the muscles deprived of nervous control [21]. The aims of the restoration vary with the 
ambition of the researchers and range from assistance with wheelchair transfers to the ability to stand up and sit down, to take few steps, to walking for some distance. Most work has concentrated on the correction of foot drop in hemiplegia and on the restoration of standing and walking in paraplegia. Yet, rapid fatigue of the artificially stimulated muscle and multiple degrees of freedom (DoF) of the lower limb emanating from the multi-planner muscle actions worsen the control of movement hindering the practical usability of the existing FES gait systems.

A hybrid orthosis system (HOS) usually combines passive mechanical braces with FES with a view to assist the FES in various ways. Besides reducing the number of degrees of freedom that otherwise have to be controlled by FES, the mechanical brace can also serve to eliminate continuous stimulation for prolonged standing, to support body weight, and to protect the joint and ligament by restricting the range of motion of the joint. The hip guidance orthosis (HGO) [27], a kind of trunk-hip-knee-ankle-foot orthosis (THKAFO), constraints all the lower extremity motion except for limited sagittal hip rotation and hence is just one strong candidate for such passive orthosis. Similar other passive orthoses include the reciprocating gait orthosis (RGO) $[5,19,24,29]$ reciprocally coupling the hip extension with the contralateral hip flexion. This arrangement of the hip mechanism passively prevents bilateral hip rotation in the same direction and thus improving the anterior trunk tilt commonly associated with FES only gait. An HOS version of the RGO incorporating FES was found to improve the issue of anterior trunk tilt further [23]. Another recent approach of HOS entails exploiting the passive mechanism to reduce the number of muscles to be stimulated. For instance, the controlled brake orthosis (CBO) $[13,14]$ incorporates magnetic particle brakes at the hip and knee joints to refine the sagittal limb dynamics driven by FES. A hybrid orthotic approach by Greene and Granat [15] used a cam-slider mechanism to transfer energy from knee to ankle during the knee flexion to produce ankle dorsiflexion during a particular portion of the swing phase. The result was a better swing foot clearance produced (along with hip and knee flexion) solely by evoking the flexion withdrawal reflex. A conceptual design of an energy storing orthosis (ESO) developed by Durfee and Rivard [6] employs a pneumatic system to harness and transfer excess energy from knee extension to facilitate ipsilateral hip extension during stance. To et al. [32] developed a hydraulic based variable constraint hip mechanism (VCHM) that can lock, free, or couple the hips on demand, thereby constraining the joints when support is needed while allowing for free motion when the hips are mobilized by FES. The controller of the VCHM is designed to modulate the hip constraints depending on the phase of gait [20]. The hip joints are coupled during double stance and locked against flexion during single stance to prevent anterior trunk tilt. During swing, the hip joints are freed to move passively or powered by FES, allowing the forward progression through leg swing. Audu et al. [2] studied the effects of the VCHM on the kinematics and kinetics of normal gait with able-bodied volunteers and compared its performance to a commercially available Isocentric RGO (IRGO). The results showed superior gait kinematics with VCHM with controlled joint coupling with a smooth and uninhibited control of hip joints.

Spring brake orthosis (SBO), a kind of HOS, combines mechanical braces (with coordinated joint locking mechanism) with an energy storage element mounted on it and FES to generate the swing phase of paraplegic gait [11]. The energy storage element; a torsion spring; is mounted on the knee joint and stores energy during the later part of the swing phase which entails knee extension through FES of quadriceps. The stored mechanical energy is then released to produce knee flexion in the following swing phase. The resultant swing phase in this case largely comprises natural oscillation of the leg segments under the influence of the spring. Consequently the trajectory thus generated would almost entirely be defined by the combined passive properties, i.e. the spring parameters and the joint passive properties at the lower limb, especially at the knee joint. This paper focuses only on determining suitable spring for the SBO through obtaining optimal spring parameters using stochastic search techniques; genetic algorithms (GAs).

\section{The spring brake orthosis concept}

Being one of the strongest muscles in the human body, the knee extensor, when artificially stimulated, possesses the potential to produce much more torque than is required just to extend the swing knee [3]. During knee extension of swing phase, SBO exploits this feature of the quadriceps through partially storing FES generated quadriceps force as potential energy in a torsion spring attached to the knee joint (see Fig. 1). A 


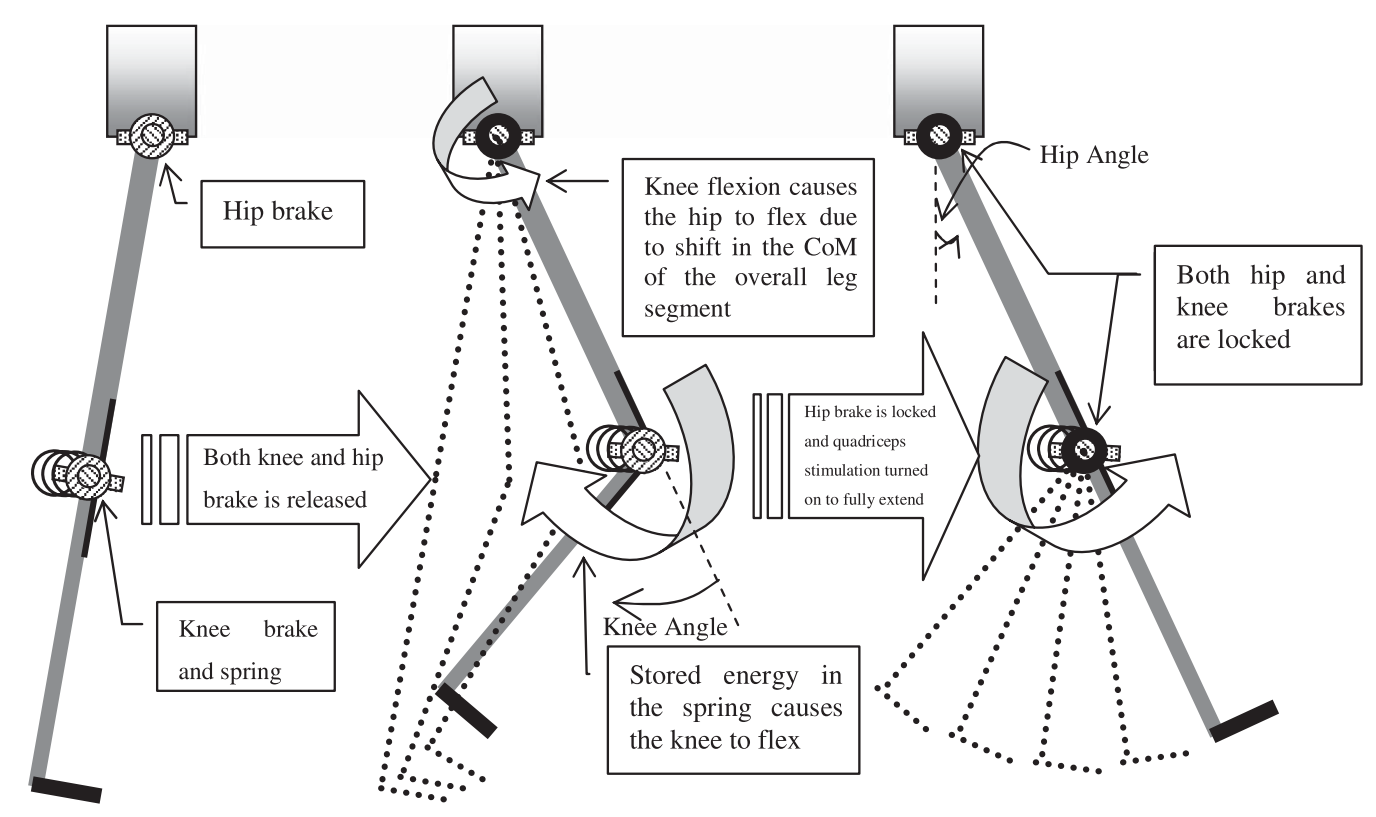

Fig. 1. Schematic of SBO concept.

brake is then employed to maintain the knee extension without any muscle contraction. The following gait cycle is then initiated through the knee flexion, achieved by releasing the brake and hence the potential energy stored in it during the FES powered knee extension during the previous gait cycle. Consequently the spring tends to return to its resting position (approx $7^{\circ}$ $-80^{\circ}$ causing the knee to flex. The hip flexion is simultaneously produced as a result of consequent shift in the centre of mass $(\mathrm{CoM})$ of the overall leg segment during this knee flexion and is maintained throughout the required duration by applying a brake/ratchet at the hip joint. This results in an HOS; the SBO, combining electrical stimulation of quadriceps muscle, spring and brake at the knee joint and brake/ratchet at the hip joint, with the activation of each of them at appropriate instants and for appropriate periods [11]. Bearing in mind that an acceptable gait can be achieved with a locked ankle [13], the ankle joint is kept fixed with an ankle-foot-orthosis (AFO).

\section{Statement of problem}

One of the major attributes of FES or HOS is the fact that it uses muscle's metabolic energy as the main source of power. Since the muscles are unidirectional, non-linear, time-varying with significant power and energy limitations, careful/optimal design is supposed to play a very important role in the degree of success in the application of an HOS. This optimal design requirement is also imposed by other factors related to successful gait generation, e.g. (a) joint range of motion, (b) speed of gait (c) controllable speed of paraplegic gait, etc.

A complete design of SBO would usually involve two distinct stages. The first one being the determination of appropriate spring parameters, i.e. 'spring constant' and its 'natural angle' for the case of conventional torsion spring; whereas the second one would comprise developing a control strategy to drive the knee joint back to full extension through FES of quadriceps. This paper particularly deals with the earlier stage of the design process. The best spring for SBO is proposed through verifying a hypothesis (Section 3.1) by intensive use of GAs. The rest of the design process, i.e. the control of FES induced movement is basically a continuation of this work and is presented as a separate paper as 'Part II'.

Although an appropriately chosen control strategy would normally be expected to be able to drive the knee joint back to full extension through quadriceps stimulation, however, simulations show that the knee as well as hip joint trajectories thus traversed are almost entirely defined just by the combination of passive dynamics of the SBO spring, joint passive viscoelasticity and leg 
segments, of which the SBO spring parameters are the only intervenable quantities. This factor renders the spring choice of particular importance in SBO design.

This particular design aspect of the SBO is formulated as an optimization problem using stochastic optimization/search techniques with a view to search for the optimal spring. Optimality is defined in context of discreetly chosen criteria specific to the swing phase of SBO. Such solutions are often sought through the solution of an appropriately formulated nonlinear programming problem. However, this approach requires the precise expression of a, usually not well understood, set of weights and goals, which, for the current problem is not available. Evolutionary algorithms (EAs), on the other hand, do not require derivative information or a formal initial estimate of the solution region. Because of the stochastic nature of the search mechanism, EAs are capable of searching the entire solution space with more likelihood of finding the global optimum than conventional optimisation methods. Indeed, conventional methods usually require the objective function to be well behaved, whereas the generational nature of EAs can tolerate noisy, discontinuous and time-varying function evaluations [12]. Under the circumstance, GAs, a kind of EAs seem to be potentially strong tools to serve the purpose.

\subsection{Conventional vs. constant torque type spring for SBO: A hypothesis}

In SBO arrangement, the flexion movement of the knee is basically part of an entirely passive resonant oscillation of the spring mass system consisting of the SBO spring, natural elasticity at the knee joint and contributing mass from the leg segments. The combination may approximately be viewed as a simple spring mass system obeying Hook's law and constituting a resonance system, whose oscillation produces a knee flexion peak at a certain instant. The equivalent spring constant including the SBO spring would thus always be higher than the spring constant of the original elasticity (natural elasticity of the knee) as the SBO spring acts in parallel to it leading to higher oscillating frequency of the mass spring system given by:

$$
\omega=\sqrt{\frac{k}{m}}
$$

where $k$ is the spring constant and $m$ is the mass.
It may be noted that, the resonance frequency of oscillation of the spring mass system would remain unaltered if it was acted upon by a constant force rather than another spring mounted in parallel. As can be seen in equation (1), a constant force in such springmass system would only contribute towards changing the amplitude of oscillation (depending on the initial condition) rather than altering the equivalent joint elasticity and hence the frequency of oscillation. This means, torsion spring providing constant torque could be an alternative against rapid swing phase in SBO generated swing phase. A constant-force type spring, known as the Neg'ator, was developed in 1952 [33]. It is evident that unlike conventional springs, constanttorque springs are capable of a long deflection and maintain uniform torque throughout their entire operating region.

\section{Methods}

The simulation environment consists of Matlab with Simulink and Visual Nastran (vN4D) software, with passive viscoelastic model implemented in Simulink combined with forward simulation of motion in vN4D.

vN4D is a commercial CAD based kinetic-dynamic simulator, which provides an easy way to perform finite element analysis (FEA) solution of rigid body systems. It offers the possibility of system movement visualization as well as interactive perturbation and modification of the system's data structure. Moreover, the graphical representations of different variables (position, velocity and acceleration) as time-functions as well as numerical data of the graphically represented variable are available. The fact that a vN4D offers the possibility of complete control and integration with Matlab/Simulink, creates a versatile environment for such forward dynamic simulation of movement with the potential of investigating numerous model as well as control features. This feature makes it especially suitable for optimization routings running within Matlab, for cases where the model used to evaluate the objective function(s) is a vN4D plant or has a vN4D plant as a part, as in the current work.

The segmental dynamics of a hanging human leg (presumably from pelvis) implemented within vN4D accounts for the forward dynamic solution, as well as visualization of motion, and is integrated with passive viscoelastic models for all the joints implemented within Simulink. The model made up of Simulink and 
vN4D plant is then used to evaluate the objective functions required by the optimization routine running in Matlab.

\subsection{The leg model}

\subsubsection{Segmental dynamics}

A link segment based model of a human leg of an average-sized person ( $75 \mathrm{~kg}$ mass and $177 \mathrm{~cm}$ height) restrained to the sagittal plane, is developed within vN4D software for the purposes of forward dynamic simulation as well as visualization of motion. The thigh, shank and foot are implemented as 3 rigid bodies. As in the originally proposed SBO the ankle joint is restrained with ankle-foot-orthosis (AFO), in the vN4D model, the shank and foot segments are connected through a 'rigid joint' to effectively act like a single rigid body. The resulting 2 rigid segments (thigh and shank-foot) interact through the 'knee joint', which is implemented as a hinge joint in the sagittal plane. This whole leg segment connects to and suspends from a fixed segment (presumably pelvis) through 'hip joint' simulated as another hinge joint in the sagittal plane. This suspension mechanism of the whole leg from hip down is due to the sole purpose of this model being the simulation of swing phase, during which the leg can be said to be suspended from the pelvis. The resultant link segment model for the human leg thus contains only 2 DoF. Anthropometric data for the segments viz. volume, mass, CoM, joint centre and moment of inertia were obtained from statistical tables based on person's height and weight [37]. Figure 2 shows the simplified schematic of the model.

\subsubsection{Passive components model}

SBO generated swing phase, comprising largely of passive oscillation, would considerably be affected by the passive viscoelastic torques at the hip and knee joints. To take this into account, the passive knee and hip joint properties model, developed by Amankwah et al. [1] is also implemented in the Simulink and incorporated with the basic leg model described above. The authors developed a model based on Kelvin model for viscoelasticity, describing the lower limb passive joint moments (ankle, knee and hip), to investigate and compare these moments amongst able bodied and disabled subjects. It consists of a nonlinear elastic element in parallel with both a linear elastic element and a nonlinear viscous element in series (Fig. 3). All the parameters required by the model equations were estimated for a number of subjects both able-bodied and paraplegic.

\subsubsection{The SBO model}

Within the vN4D software environment there are spring and brake placed at the knee joint. At the hip joint, a ratchet is implemented which, if required, holds the joint at its maximum flexion angle. All these can be controlled and parameterized from the Simulink environment, and hence Matlab. The extra weight incurred by the orthosis would obviously affect the segmental dynamics. This is approximately accounted for by an extra $2.25 \mathrm{~kg}$ weight uniformly added to the leg segments [31]. The joint kinematics and the associated moments are expressed according to the convention considered in Riener and Fuhr [26] and is presented in Fig. 4.

\subsection{The search operation}

\subsubsection{Genetic algorithms}

First proposed by Holland [17], GAs are search and optimisation techniques inspired by two biological principles of natural evolution, viz. the process of 'natural selection' and the mechanics of 'natural genetics'. GAs have emerged as useful searching method in recent years $[10,12,30]$. Contrary to conventional search algorithms which aim for one single solution,

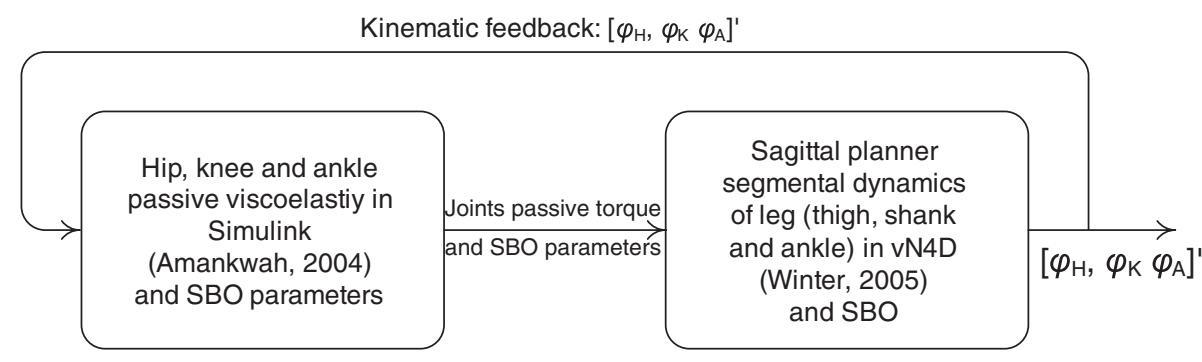

Fig. 2. Schematic of the leg model. $\varphi_{\mathrm{H}}, \varphi_{\mathrm{K}}$ and $\varphi_{\mathrm{A}}$ are hip, knee and ankle angles respectively. 


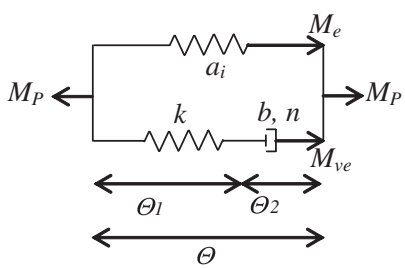

Fig. 3. Mechanical analogue of passive moment model. Two elastic elements are represented with model parameters $k$ and $a_{\mathrm{i}}$, while parameters $b$ and $n$ characterize viscous element. Joint angle is represented by $\Theta$, and $M_{e}, M_{v e}$ and $M_{P}$ are nonlinear passive elastic moment, viscoelastic moment and passive moment developed at joint respectively, from [1].

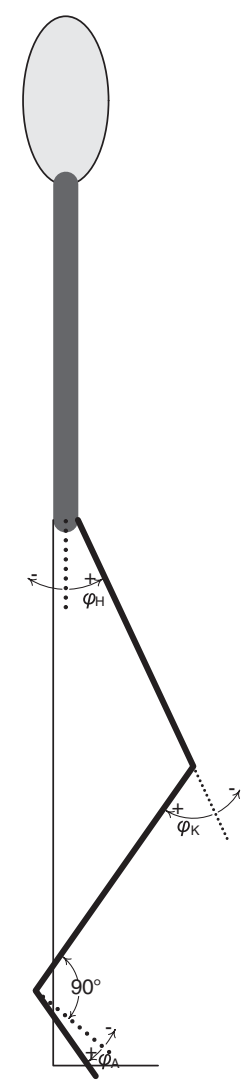

Fig. 4. The joint kinematic convention followed in this work. $\varphi_{\mathrm{H}}$, $\varphi_{\mathrm{K}}$ and $\varphi_{\mathrm{A}}$ are hip-, knee- and ankle-angle respectively.

GAs manipulate a collection of potential solutions, called a population. The potential solutions in the population, called "individuals" or "chromosomes", are encoded representations of all the parameters of the solution. Each chromosome is assigned a fitness rating according to its success compared to the other chromosomes in the population. 'Evolution' of the chromosomes that encode better solutions is achieved through the use of so-called "genetic operators", such as crossover and mutation, creating new chromosomes by either merging two or more original (parent) chromosomes or by modifying an existing chromosome. The selection mechanism for parent chromosomes takes the fitness of the parents into account, ensuring that the better solutions have a higher chance to procreate and donate their beneficial characteristics to their offspring. When ready, newly generated individuals replace the existing ones creating a better population. The population converges to a 'best' solution after a number of iterations [34].

\subsubsection{Multi-objective optimization and multi-objective GAs}

Real world problems, in most cases, involve multiple objectives to be achieved simultaneously. Due to the conflicting nature of the objectives, it is often difficult to find a single optimal solution for a problem, and it makes more sense to seek a set of nondominated Perato-optimal solutions for which an improvement in one of the objectives will lead to degradation in one or more of the remaining objectives [4]. Conventionally, there are a number of approaches including the e-constraint, weighted sum and goal attainment methods that have widely been used both with nonlinear programming and EAs to deal with such multiobjective scenario [18]. Like many others, these approaches also require precise expressions of weights and goals. Also the aggregation function to combine the objectives may need to be tuned by repeated run of the optimizer.

EAs are suitable techniques for multi-objective optimisation due to their population based nature which enables them to support multiple solutions concurrently. Finding multiple nondominated solutions by treating objectives separately was first initiated by Schaffer [28]. Other approaches exploiting EAs with a view to search simultaneously for multiple nondominated solutions include Fourman [9], Kursawe [22], and Hajela and Lin [16]. However, none of these makes direct use of the actual definition of Pareto-optimality [4] and different non-dominated individuals are generally assigned different fitness values.

Pareto-based approaches on the other hand guarantee equal probability of reproduction of all nondominated solutions. One of the first approaches to utilise the concept of Pareto optimality in GAs was 


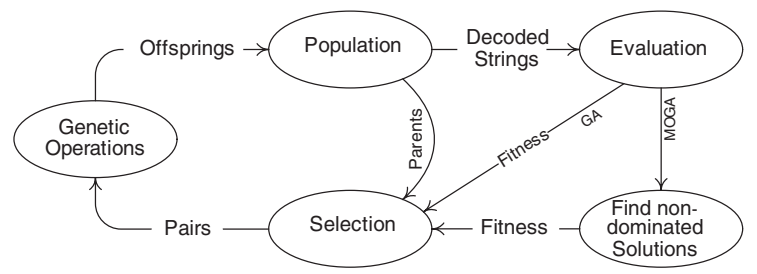

Fig. 5. GA and MOGA reproduction cycle.

Fonseca and Fleming's multi-objective GA (MOGA) [8]. MOGA differs from standard GA in the way fitness is assigned to each solution in the population. The rest of the algorithm (the stochastic universal sampling, a single-point crossover, and a bit-wise mutation) is the same as that in a classical GA. A simplified schematic of the GA and MOGA reproduction cycle is presented in Fig. 5.

\subsubsection{Choice of objective function}

The first factor that influences the choice of the objective function is that the larger (than normal) swing phase required for the control task to be easier. Larger duration of swing phase results in a slower swing phase and hence a slower gait cycle is preferable to make the control operation easier [13]. Accordingly, the search operation is performed to result in spring parameters that will generate a swing phase of longer in duration than that of normal gait ( 0.55 second).

One of the key features of SBO is to implement the swing phase through passive oscillation as much as possible. This would act towards increasing energy efficiency, reducing the burden on the controller and increasing repeatability. This is implemented by designing the associated control system so that it triggers the quadriceps stimulation (to drive the knee joint back to full extension) just before the shank starts to oscillate back towards flexion. In this study, the particular instant of passive oscillation (at the end of one full cycle, when the knee joint attains zero angular velocity) is considered as the end of potential swing phase and roughly defined as the duration of the generated swing phase. Its inverse is defined as one of the objective functions to be minimized in this study.

The peak knee flexion occurring during the swing phase would hold some sort of relationship with the swing duration, and must be taken care of. The peak knee flexion in natural gait is approximately $65^{\circ}$ [36], and so it would be desirable to keep it close to that value in the SBO aided gait, while stretching the swing duration as much as possible. This additional goal asks, in optimization terms, for a second objective to be formulated. This makes the optimization problem a multi-objective one with the deviation of the peak knee flexion from $65^{\circ}$ as an additional objective.

\section{Results}

\subsection{Optimization of the SBO spring parameters: Conventional spring}

Initial effort is focussed on producing the swing phase knee flexion peak at a particular point in time and flexion angle. A GA with a population size of 20 individuals is run 4 times, each time for 100 generations, to find the best spring parameters, i.e. 'spring constant' and 'natural angle' so that the SBO aided knee flexion peak corresponds to those points, i.e. $\left(0.31 \mathrm{sec}, 62.2^{\circ}\right.$, $\left(0.5 \mathrm{sec}, 62.2^{\circ}\right.$ and $\left(0.2 \mathrm{sec}, 70^{\circ}\right.$. The objective function $\mathrm{f}(y)$ to be minimized is thus formulated as:

$$
\mathrm{f}(\mathrm{y})=\left(\mathrm{t}_{\mathrm{p}}-t_{y}\right)^{2}+\left(\varphi_{\mathrm{p}}-\varphi_{\mathrm{y}}\right)^{2}
$$

where $\left(t_{p}, \varphi_{p}\right)$ and $\left(t_{y}, \varphi_{y}\right)$ are the coordinates (in the 'time' vs. 'angle' plane) of the fixed target point ' $p$ ' and the actual knee flexion peak respectively.

Figure 6 shows the resulting knee and corresponding hip joint trajectories. The desired knee flexion peak points are represented by six pointed stars in the figure, the biggest star being the one corresponding to natural gait. The figure also shows the knee joint trajectory purely under the influence of passive moments developed by [1] (marked by + ). In other words, this would be the actual trajectory that the knee joint would traverse through if the paraplegic leg were released from a stretched position (i.e. $0^{\circ}$ knee joint angle and $-3.7^{\circ}$ hip joint angle) absolutely without any external influence whatsoever.

The trajectory traversed by the passive knee joint clearly manifests the influence of elastic part of the passive property of the knee joint, which is very similar in effect to the spring connected in SBO. It is also worth noting that the peak of the trajectory occurs approximately at 0.3 second, which happens to be the same as that of the natural gait (bold solid line). This could be considered as the natural oscillation caused by an equivalent spring and obviously strengthens the fact that the motion of swing leg is often linked 


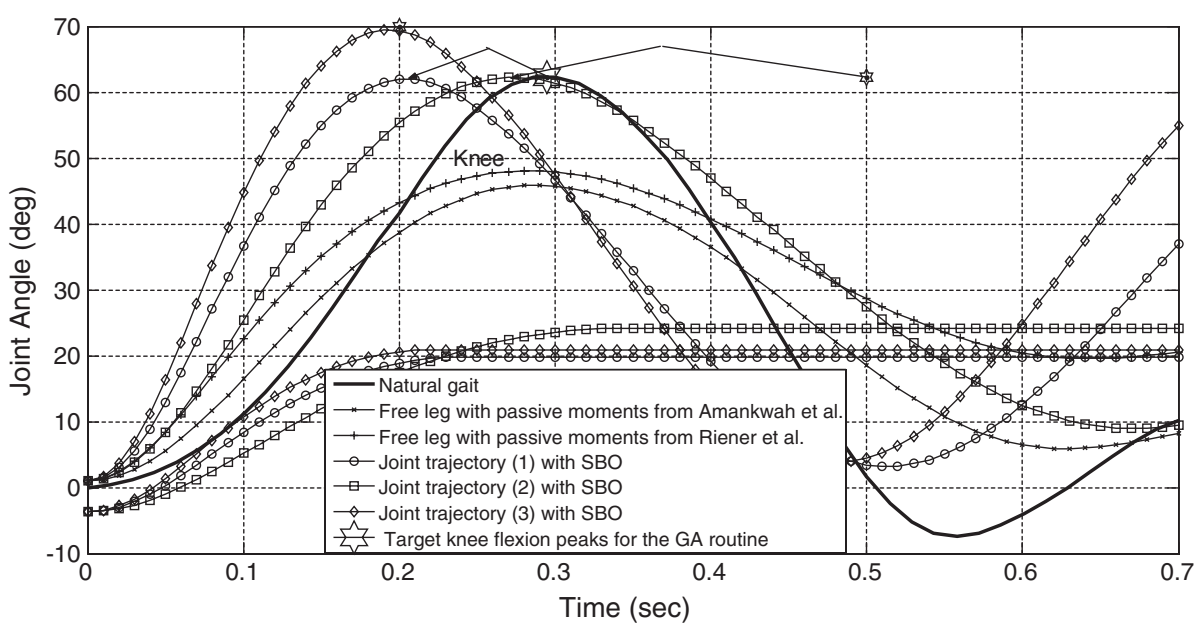

Fig. 6. Knee and hip joint trajectories 1,2 and 3 with spring parameters optimized for the knee flexion peaks to appear at $\left(0.31\right.$ sec, $62.2^{\circ}$, $\left(0.5 \mathrm{sec}, 62.2^{\circ}\right.$ and $\left(0.2 \mathrm{sec}, 70^{\circ}\right.$ respectively. The arrowed lines indicate the distances of the actual knee flexion peaks from the target points as used in the GA routine.

to the unforced swinging of a compound pendulum [25].

The optimization procedure is primarily aimed to search for the spring parameters to produce the knee flexion peak at an instant beyond 0.3 second, so as to stretch the duration of the swing phase. No special attention is paid in choosing the corresponding magnitudes of the flexion peaks, and these are chosen just arbitrarily. While the solutions were quite acceptable for cases with target knee flexion peaks appearing on the left hand plane of $X=0.3$ second line, i.e. $(0.2 \mathrm{sec}$, $70^{\circ}$, the GA optimization procedure apparently fails to come up with solutions for cases where the target knee flexion peaks appear on the right hand plane of that line, which ultimately implies inability to stretch the swing duration beyond that of the normal gait. Moreover, not only it seems to be impossible to produce a delayed knee flexion peak just with a passive spring, rather it speeds it up.

Further investigation was carried out with the same GA routine but with higher values of target knee flexion peaks, $\left(0.31 \mathrm{sec}, 75^{\circ}\right.$ and $\left(0.5 \mathrm{sec}, 90^{\circ}\right.$, with the same objective function as defined in equation (2) and the result is shown in Fig. 7. Apparently higher knee

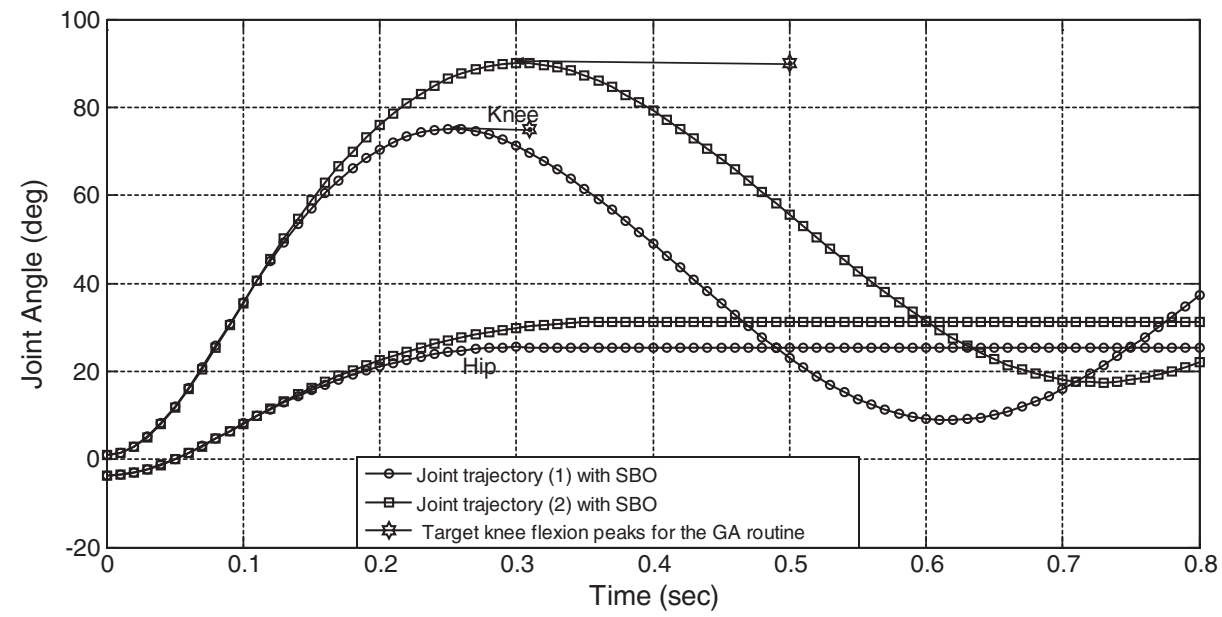

Fig. 7. Knee and hip joint trajectories (1) and (2) with spring parameters optimized for higher target knee flexion peaks $\left(0.31\right.$ sec, $\left.75^{\circ}\right)$ and $\left(0.5 \mathrm{sec}, 90^{\circ}\right.$ respectively. The arrowed lines indicate the distances of the actual knee flexion peaks from the target points as used in the GA routine. 


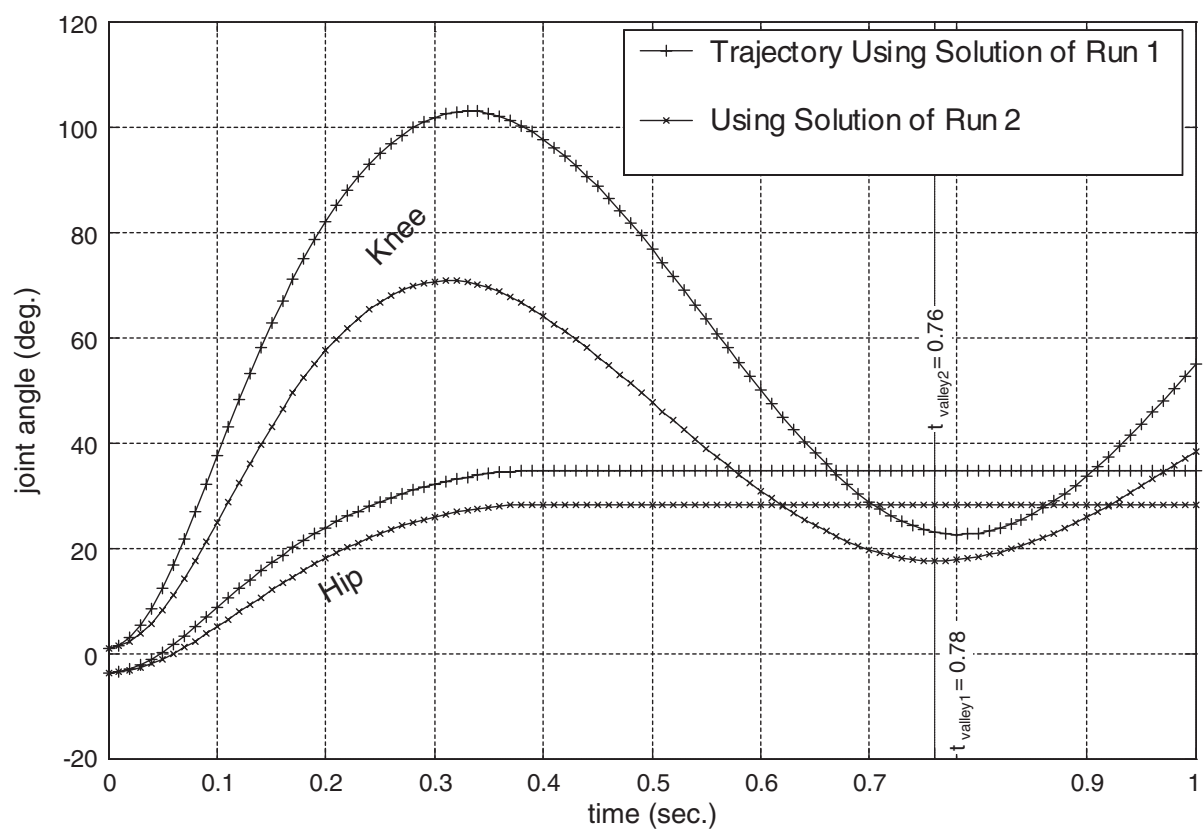

Fig. 8. Knee and hip joint trajectories with spring parameters optimized for maximum possible swing duration.

flexion angles were found to be able to stretch the swing phase to up to $0.72 \mathrm{sec}$, while the peak still appeared within 0.3 second. This asymmetry between the halves of the swing phase can be accounted for partly by the hip joint being locked halfway during the swing phase resulting in different segmental dynamics in the two halves of the swing phase; and partly by the nonlinearity of the knee joint passive viscoelastic properties.

As a straightforward approach, spring parameters were optimized for maximum possible swing duration. This was achieved by setting the inverse of the potential swing duration as the objective function $\mathrm{f}(y)$ to be minimized.

$$
\mathrm{f}(y)=\frac{1}{\left(t_{\text {valley }}\right)}
$$

where $t_{\text {valley }}$ is the time instant of the occurrence of the extension peak following the flexion peak.

The results of two individual runs are shown in Fig. 8. It is evident from this result that the swing duration is eccentrically related to the magnitude of the peak knee flexion. Although the first run of the optimization process could stretch the swing duration to $0.78 \mathrm{sec}$, but only at the cost of a higher knee flexion $\left(>100^{\circ}\right)$; the second run also stretched the swing duration to a similar amount along with a near natural knee flexion peak $\left(\right.$ at $\left.70^{\circ}\right)$.
Such eccentric relation between the peak knee flexion and the overall swing duration suggests that these two features might need to be dealt with individually, forming two individual objective functions with a multi-objective optimization tool. The first objective, $\mathrm{f}_{1}(y)$ was defined as the inverse of the overall swing duration [equation (3)] while a second objective, $f_{2}(y)$ was the absolute value of the deviation of the knee flexion peak from $65^{\circ}$ as:

$$
\mathrm{f}_{2}(y)=\left|65-\varphi_{\text {peak }}\right|
$$

where $\varphi_{\text {peak }}$ is the peak knee flexion angle in degrees attained by the resultant knee trajectory.

A MOGA with a population size of 20 binary coded individuals was employed to this multi-objective optimization problem twice, each time for 100 generations, which achieved 6 Pareto-optimal solutions.

The complete result of employing MOGA is shown in Fig. 9, which shows the set of Pareto-optimal solutions and their corresponding parameter values and also the knee and hip joint trajectories corresponding to two of the solutions.

The results simply re-establish the fact that it is virtually impossible to stretch the SBO swing duration beyond 0.75 second (trajectory with minimum value of $\mathrm{f}_{2}(y)$ corresponding to solution 6 ). The set of Pareto-optimal solutions exhibits a very little variation 


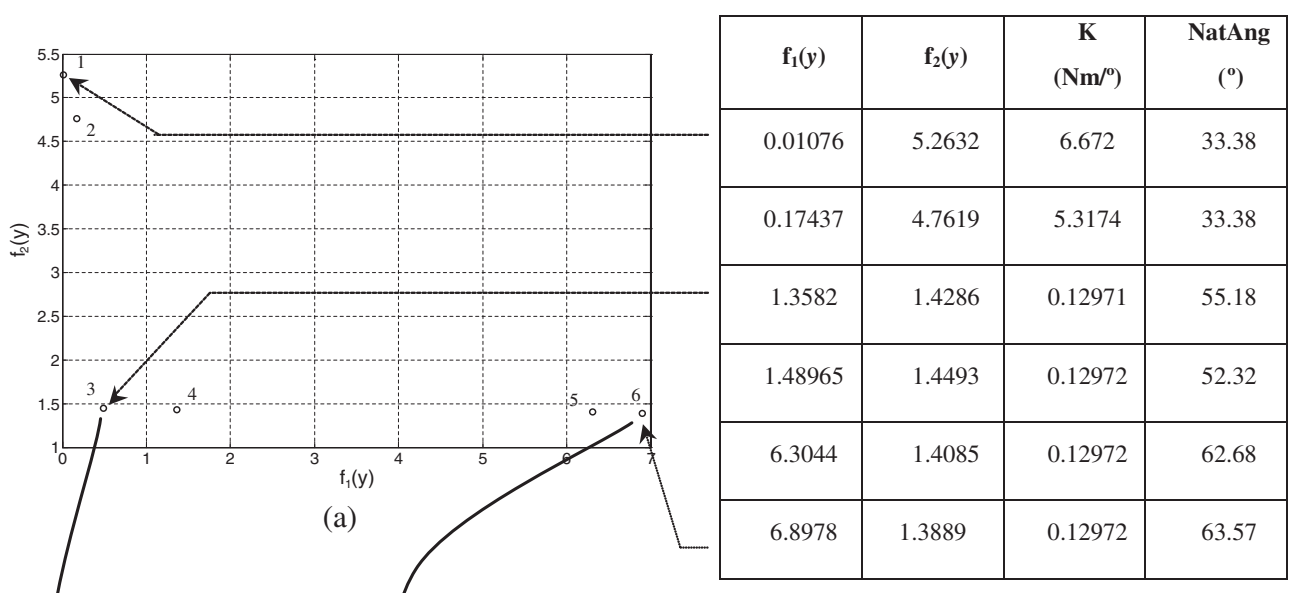

(b)

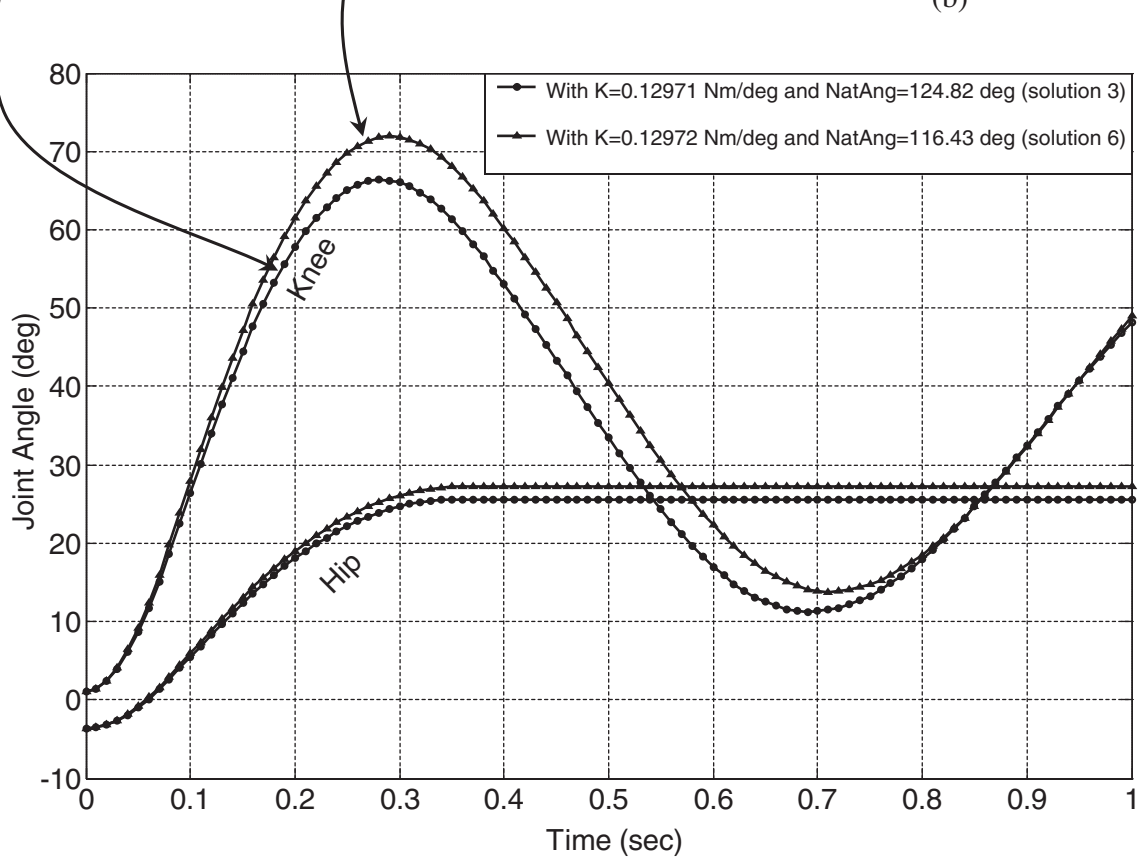

(c)

Fig. 9. MOGA optimization (minimization problem) of spring parameters (a) Set of Pareto-optimal solutions (b) Table containing the set of Pareto-optimal solutions with the corresponding spring parameter values (c) Limb joint trajectories corresponding to some Pareto-optimal solutions.

of approximately $10 \%$ (of $65^{\circ}$ ) in the $\mathrm{f}_{1}(y)$, whereas variation in $\mathrm{f}_{2}(y)$ is approximately $279 \%$ (of its best value). But, if solutions 1 and 2 are excluded, this variation comes down to $\approx 2.86 \%$ for solutions $3-6$. Thus, the 4 Pareto-optimal solutions 3-6 do not really encompass a broad range, indicating a less conflicting nature of the objectives. In other words, this result can be summarized as providing an SBO spring that generates a swing phase with a knee flexion peak very near to $67^{\circ}$ and with duration of approximately 0.75 second. This result also indicates that maximum possible SBO aided swing duration corresponds very much to a peak knee flexion angle of approximately $65^{\circ}$.

All the results so far also confirm how the SBO can generate an acceptable hip flexion $\left(25^{\circ}-30^{\circ}\right.$ without any external moment at the hip joint. 


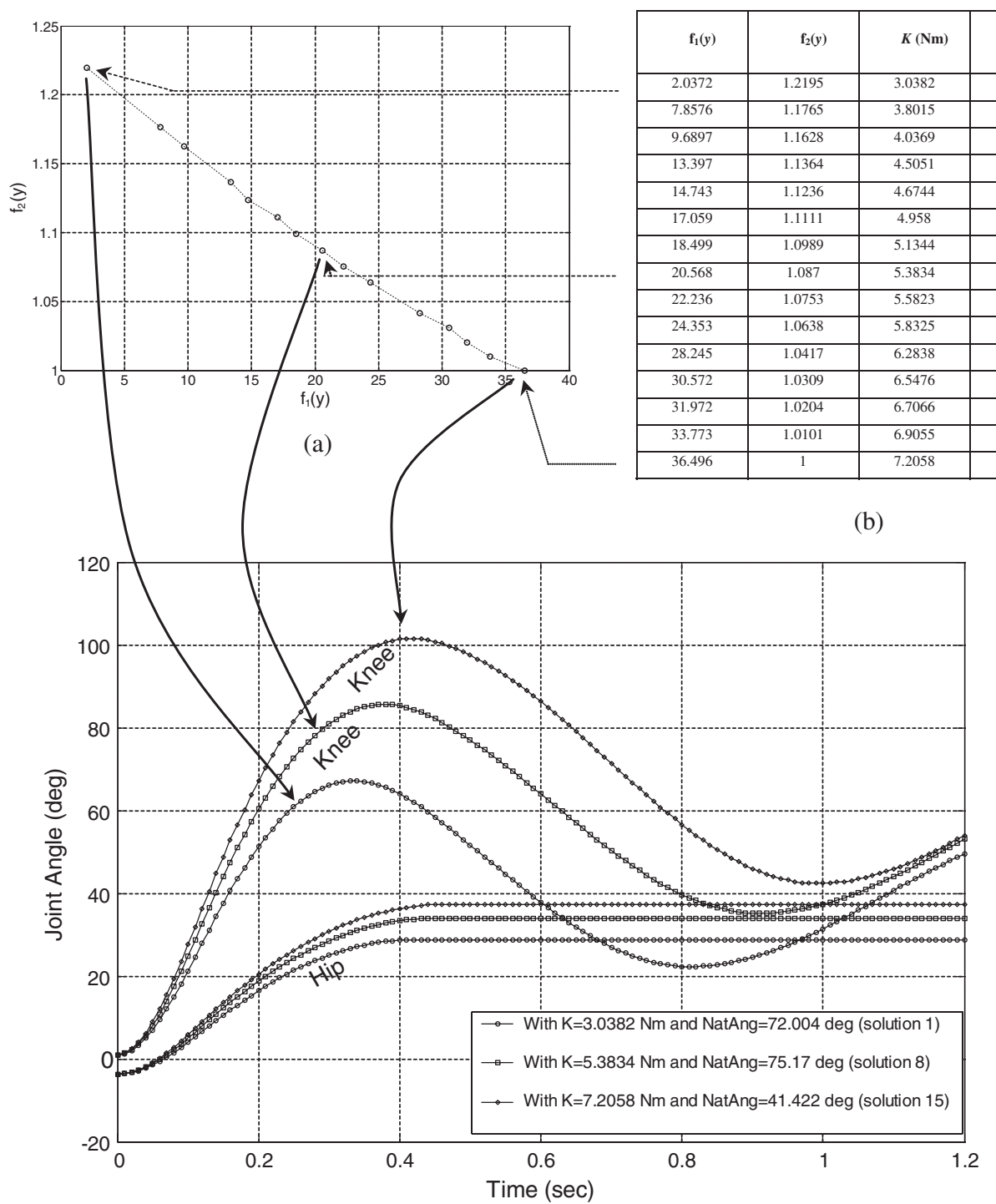

(c)

Fig. 10. Constant-torque spring parameters optimization with MOGA (a) Set of Pareto-optimal solutions (b) Table containing the set of Pareto-optimal solutions with the corresponding spring parameter values (c) Limb joint trajectories corresponding to different Pareto-optimal solutions.

\subsection{Optimization of the SBO spring parameters: Constant torque type spring}

The model used so far was modified by changing the spring of the SBO from a conventional one to a constant-torque type. The same MOGA used in the earlier section was utilised to optimize constanttorque spring parameters, viz. the constant torque $K$ $(\mathrm{Nm})$ and its natural angle $\left(^{\circ}\right)$. Once again, the absolute value of the deviation of the peak knee flexion from $65^{\circ}$ was set as first objective or $f_{1}(y)$ [equation (3)], while the inverse of the swing duration was set as a second objective or $f_{2}(y)$ [equation (4)]. The result (Fig. 10) shows the Pareto-optimal front consisting of 15 nondominated solutions, along with the corresponding parameter values and some resultant trajectories.

The result seemed to be quite an improvement in terms of increasing the swing duration and thus exactly in line with the hypothesis of SBO spring being combined in parallel with the knee joint 
elasticity. According to the hypothesis, constant torque spring was supposed to increase the swing duration (compared to that of normal spring), and it is evident from equation (1) that even the lowest swing duration available from the solutions is more than 0.8 second, which was not possible to achieve with normal spring. Also unlike the normal spring case, the objectives seem to hold a rather straightforward linear relationship (Fig. 10a); higher knee flexion peak produces longer swing phase. The solution set also occupies a broader range and is less localized compared to normal spring case. $\mathrm{f}_{1}(y)$ and $\mathrm{f}_{2}(y)$ have variations of $53 \%$ (of $65^{\circ}$ ) and $22 \%$ (of its best value) respectively, which are much higher than the normal spring case (10\% and $2.86 \%$ respectively).

\subsection{Comparison between constant-torque spring and conventional spring}

For the sake of a quantitative comparison between the performances of the two spring types, the Paretooptimal solution sets for both are placed in the same plot in Fig. 11. As can be seen in the figure, the Pareto-optimal solution front associated with the constant-torque spring tends to lie closer to the origin indicating a better performance, especially in terms of the (elongating) swing duration. It can therefore be concluded that the normal spring is better in minimizing objective- 1 or $\mathrm{f}_{1}(y)$, i.e. keeping the knee flexion peak near $65^{\circ}$, while the constant-torque type spring performs much better in minimizing objective- 2 or $\mathrm{f}_{2}(y)$, referring to a larger swing duration. This result reveals a clear picture of the fact that the constant-

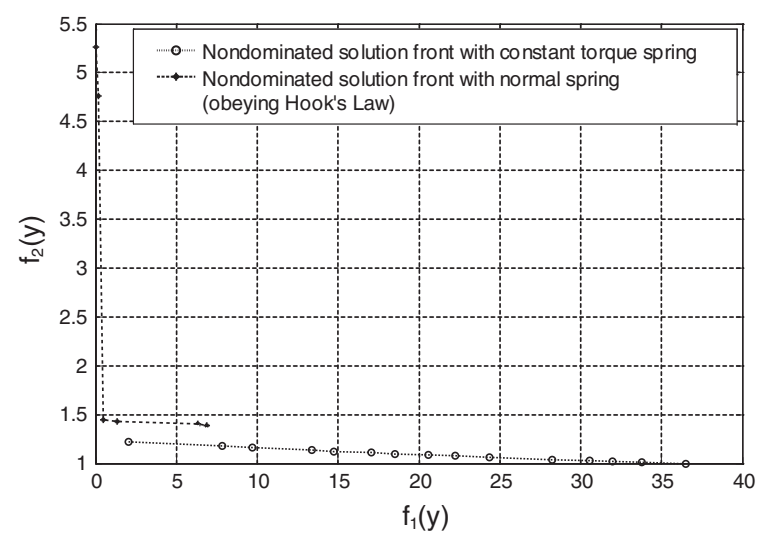

Fig. 11. Pareto-optimal solution sets with conventional spring and constant-torque spring. torque spring is capable of producing a longer SBO swing phase than that of the normal spring, in full agreement with the hypothesis made in Section 3.1.

It may also be noticed from Fig. 11 that no member of the Pareto-optimal solution set for the constanttorque spring was dominated by those of the normal spring. Two of the 6 solutions $(50 \%$ of the major 4 solutions) for the normal spring are dominated by those of the constant-torque spring, which clearly asserts the superiority in performance of the constant-torque spring in SBO assisted swing phase.

\section{Discussion}

Initially the study searches for conventional torsion spring that could produce longer (than normal) swing duration to provide an easier to control slower swing phase [6]. During the process it is revealed that the SBO aided gait cannot be designed to have arbitrarily large swing duration; a fact supporting the authors' hypothesis. Consequent searches were carried out step-by-step with a view to confront this limitation. Although initially it was intended to relate the knee flexion peak with the swing duration, it was found that these two are related only eccentrically.

The very limited duration of the possible swing duration has the potential to pose an important challenge from the control perspective of SBO gait [13]. Constant torque type spring, instead of originally proposed conventional torsion spring has been found to circumvent the problem to some extent. But since the objectives of (a) stretching the swing duration and $(b)$ keeping knee flexion peak close to $65^{\circ}$ was found to be conflicting. Thus, careful design considerations have to be made in practice to deal with such trade off situation. Due to the conflicting nature of the objective functions, the best spring parameters; i.e. the output of the MOGA is basically a set of Pareto optimal solutions (Fig. 10b) and a designer can pick any of these solutions using expert knowledge, perhaps depending on the rest of the gait cycle.

From practical design point of view, the constanttorque spring may provide additional advantage over the conventional torsion spring. Since the constanttorque is a combination of a constant-force spring and an output drum, virtually any constant torque spring of arbitrary specification can easily be constructed from a constant force spring of a fixed type, simply by choosing the output drum of an appropriate 
diameter. In practical terms, this means if a pool of a fixed type of constant force spring and output drum of a range of diameter is maintained, it is possible to realize the required constant-torque spring for a specific subject without much trouble. Torsion spring of specific parameters (for a specific subject), on the other hand, would require ordering to the manufacturers with the required specifications. Even if a pool is maintained, it is supposed to hold springs of a range of both spring constants and natural angles.

\section{Conclusions}

Careful optimization and hence energy efficiency ought to play a vital role in the degree of success of an HOS, since muscle's metabolic energy is the main source of energy to drive such a system. Stochastic search techniques such as GAs can be successfully used within simulation environment in such design process prior to practical implementation, where even trial and error approach is not a great option with human subjects being involved. Constant torque spring replacing the originally proposed conventional torsion spring seems to be more preferred option both in terms of paraplegic gait control and accommodating wide range of subjects. The leg model used in this simulation assumes an average-sized person $(75 \mathrm{~kg}$ mass and $177 \mathrm{~cm}$ height) as the subject while such design of SBO might turn out to be highly subjective. To avoid such computational intensive design process for every individual subject, a model or function might be developed that would map some easily measurable subject specific data to appropriate spring parameters.

\section{References}

[1] K. Amankwah, R.J. Triolo and R. Kirsch, Effects of spinal cord injury on lower-limb passive joint moments revealed through a nonlinear viscoelastic model, J Rehabil Res Dev 41(1) (2004), 15-32.

[2] M.L. Audu, C.S. To, R. Kobetic and R.J. Triolo, Gait evaluation of a novel hip constraint orthosis with implication for walking in paraplegia, Ieee Transactions on Neural Systems and Rehabilitation Engineering 18(6) (2010), 610618.

[3] T. Bajd, A. Kralj, M. Stefancic and N. Lavrac, Use of functional electrical stimulation in the lower extremities of incomplete spinal cord injured patients, Artif Organs 23(5) (1999), 403-409.

[4] A. Ben-Tal, Characterization of Pareto and lexicographic optimal solutions, Springer-Verlag, Berlin, 1980, pp. 1-11.
[5] R. Douglas, P.F. Larson, R. D'Ambrosia and R.E. McCall, The LSU reciprocation gait orthosis, Orthopedic 6 (1983), 834-839.

[6] W.K. Durfee and A. Rivard, Design and simulation of a pneumatic, stored-energy, hybrid orthosis for gait restoration, J Biomech Eng 127(6) (2005), 1014-1019.

[7] I. Eltorai and R. Montroy, Muscle release in the management of spasticity in spinal cord injury, Paraplegia 28(7) (1990), 433-440.

[8] C.M. Fonseca and P.J. Fleming, Genetic algorithms for multiobjective optimisation: Formulation discussion and generalization, Fifth International Conference on Genetic Algorithms, Morgan Kaufmann, San Mateo, CA, 1993, pp. 416-423.

[9] M. Fourman, Compaction of symbolic layout using genetic algorithms, Proc International Conference on Genetic Algorithms and their Applications 1985, pp. 141-153.

[10] M. Gen and R. Cheng, Genetic algorithms and engineering optimization, Chichester, Wiley-Interscience, New York, 2000, xvi, 495 p.

[11] S. Gharooni, B. Heller and M.O. Tokhi, A new hybrid spring brake orthosis for controlling hip and knee flexion in the swing phase, IEEE Trans Neural Syst Rehabil Eng 9(1) (2001), 106-107.

[12] D.E. Goldberg, Genetic algorithms in search, optimization, and machine learning, Reading, Mass, Addison-Wesley. 1989, $412 \mathrm{p}$.

[13] M. Goldfarb and W.K. Durfee, Design of a controlled-brake orthosis for FES-aided gait, IEEE Trans Rehabil Eng 4(1) (1996), 13-24.

[14] M. Goldfarb, K. Korkowski, B. Harrold and W. Durfee, Preliminary evaluation of a controlled-brake orthosis for FESaided gait, IEEE Trans Neural Syst Rehabil Eng 11(3) (2003), 241-248.

[15] P.J. Greene and M.H. Granat, A knee and ankle flexing hybrid orthosis for paraplegic ambulation, Med Eng Phys 25(7) (2003), 539-545.

[16] P. Hajela and C.Y. Lin, Genetic search strategies in multicriterion optimal design, Structural and Multidisciplinary Optimization V4(2) (1992), 99-107.

[17] J.H. Holland, Adaptation in Natural and Artificial Systems, Ann Arbor, The University of Michigan Press, 1975, $211 \mathrm{p}$.

[18] C.L. Hwang and A.S.M. Masud, Multiple objective decision making, methods and applications : A state-of-the-art survey, Springer-Verlag, Berlin, New York, 1979, xii, 351 p.

[19] R.J. Jefferson and M.W. Whittle, Performance of three walking orthoses for the paralysed: A case study using gait analysis, Prosthet Orthot Int 14(3) (1990), 103-110.

[20] R. Kobetic, C.S. To, J.R. Schnellenberger, M.L. Audu, T.C. Bulea, R. Gaudio, G. Pinault, S. Tashman and R.J. Triolo, Development of hybrid orthosis for standing, walking, and stair climbing after spinal cord injury, Journal of Rehabilitation Research and Development 46(3) (2009), 447-462.

[21] A.R. Kralj and T. Bajd, Functional electrical stimulation: Standing and walking after spinal cord injury, Boca Raton, Fla., CRC Press, 1989, 198 p.

[22] F. Kursawe, A Variant of Evolution Strategies for Vector Optimization, Proceedings of the 1st Workshop on Parallel Problem Solving from Nature, Springer-Verlag, 1991, pp. 193-197.

[23] E.B. Marsolais, R. Kobetic, G. Polando, K. Ferguson, S. Tashman, R. Gaudio, S. Nandurkar and H.R. Lehneis, The Case 
Western Reserve University hybrid gait orthosis, J Spinal Cord Med 23(2) (2000), 100-108.

[24] W. Motloch, Principles of orthotic management for child and adult paraplegia and clinical experience with isocentric RGO, 7th World Congr Int Soc Prosthet Orthot, Chicago, IL, 1992, $28 \mathrm{p}$.

[25] S.J. Piazza and S.L. Delp, The influence of muscles on knee flexion during the swing phase of gait, J Biomech 29(6) (1996), 723-733.

[26] R. Riener and T. Fuhr, Patient-driven control of FESsupported standing up: A simulation study, IEEE Trans Rehabil Eng 6(2) (1998), 113-124.

[27] G.K. Rose, The principles and practice of hip guidance articulations, Prosthet Orthot Int 3(1) (1979), 37-43.

[28] J.D. Schaffer, Multiple objective optimization with vector evaluated genetic algorithms, 1st Int Conf Genetic Algorithms, Lawrence Erlbaum Associates, Inc., Mahwah, NJ, USA, 1985, pp. 93-100.

[29] M. Solomonow, R. Baratta, S. Hirokawa, N. Rightor, W. Walker, P. Beaudette, H. Shoji and R. D'Ambrosia, The RGO Generation II: Muscle stimulation powered orthosis as a practical walking system for thoracic paraplegics, Orthopedics 12(10) (1989), 1309-1315.

[30] M. Srinivas and L.M. Patnaik, Genetic Algorithms - a Survey, Computer 27(6) (1994), 17-26.

[31] C.S. To, R.F. Kirsch, R. Kobetic and R.J. Triolo, Simulation of a functional neuromuscular stimulation powered mechan- ical gait orthosis with coordinated joint locking, IEEE Trans Neural Syst Rehabil Eng 13(2) (2005), 227-235.

[32] C.S. To, R. Kobetic, J.R. Schnellenberger, M.L. Audu and R.J. Triolo, Design of a variable constraint hip mechanism for a hybrid neuroprosthesis to restore gait after spinal cord injury, Ieee-Asme Transactions on Mechatronics 13(2) (2008), 197-205

[33] F.A. Votta, Theory and design of long-deflection constant force spring elements, Transaction of the ASM 74 (1952), 439-450.

[34] Q. Wang, P. Spronck and R. Tracht, AN Overview of Genetic Algorithms Applied to Control Engineering Problems, Machine learning and cybernetics, ICMLC 03, Xian, China, IEEE, 2003, pp. 1-16.

[35] R.L. Waters, J.S. Yakura, R.H. Adkins and I. Sie, Recovery following complete paraplegia, Arch Phys Med Rehabil 73(9) (1992), 784-789.

[36] D.A. Winter, Biomechanics and motor control of human movement, New Yorkm, Chichester, Wiley, 1990, xvi, 277p ill $25 \mathrm{~cm}$.

[37] D.A. Winter, Biomechanics and motor control of human movement, Hoboken, New Jersey, John Wiley \& Sons, 2005 xvi, 325p.: ill.; $25 \mathrm{~cm}$.

[38] J.S. Yakura, R.L. Waters and R.H. Adkins, Changes in ambulation parameters in spinal cord injury individuals following rehabilitation, Paraplegia 28(6) (1990), 364-370. 

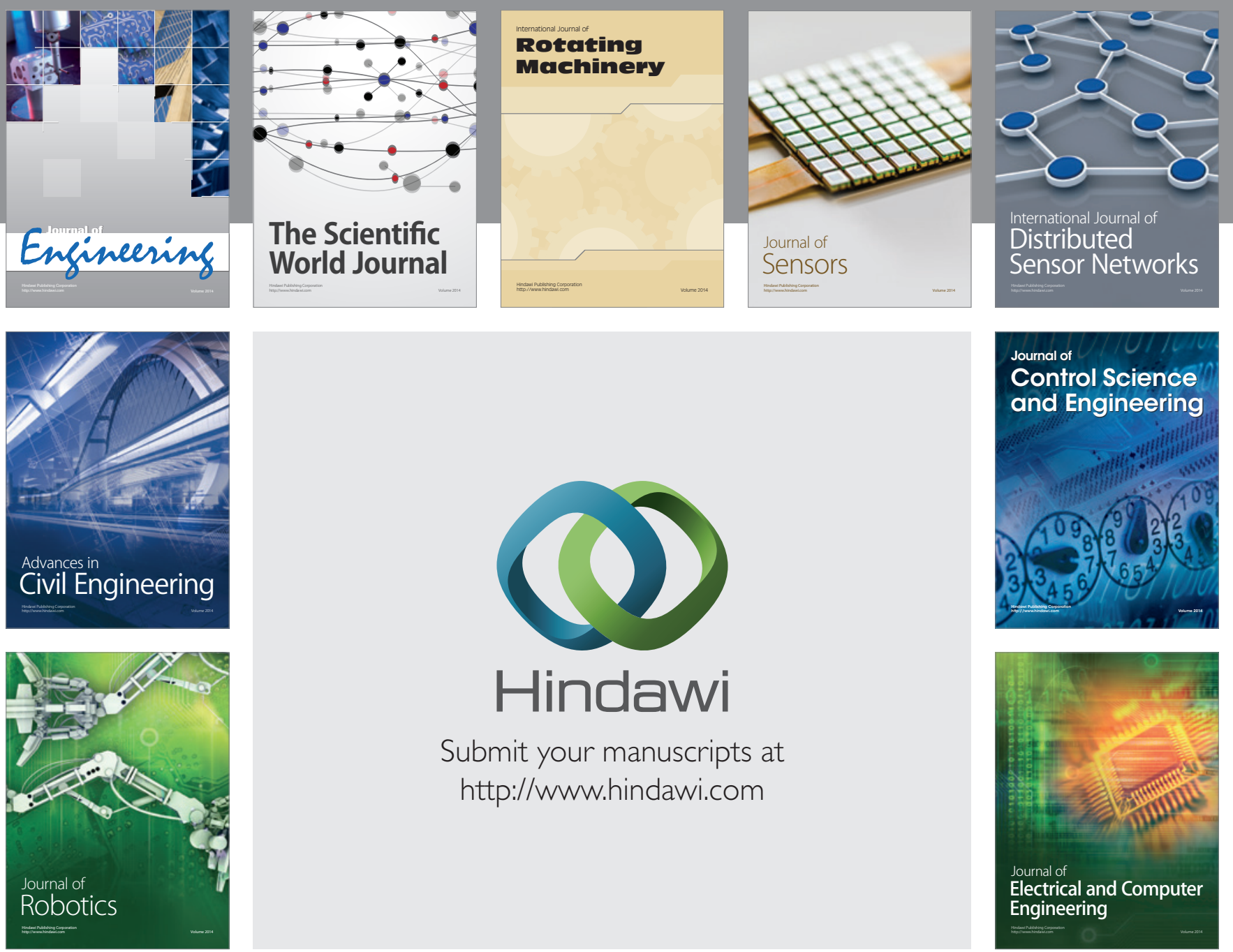

Submit your manuscripts at

http://www.hindawi.com
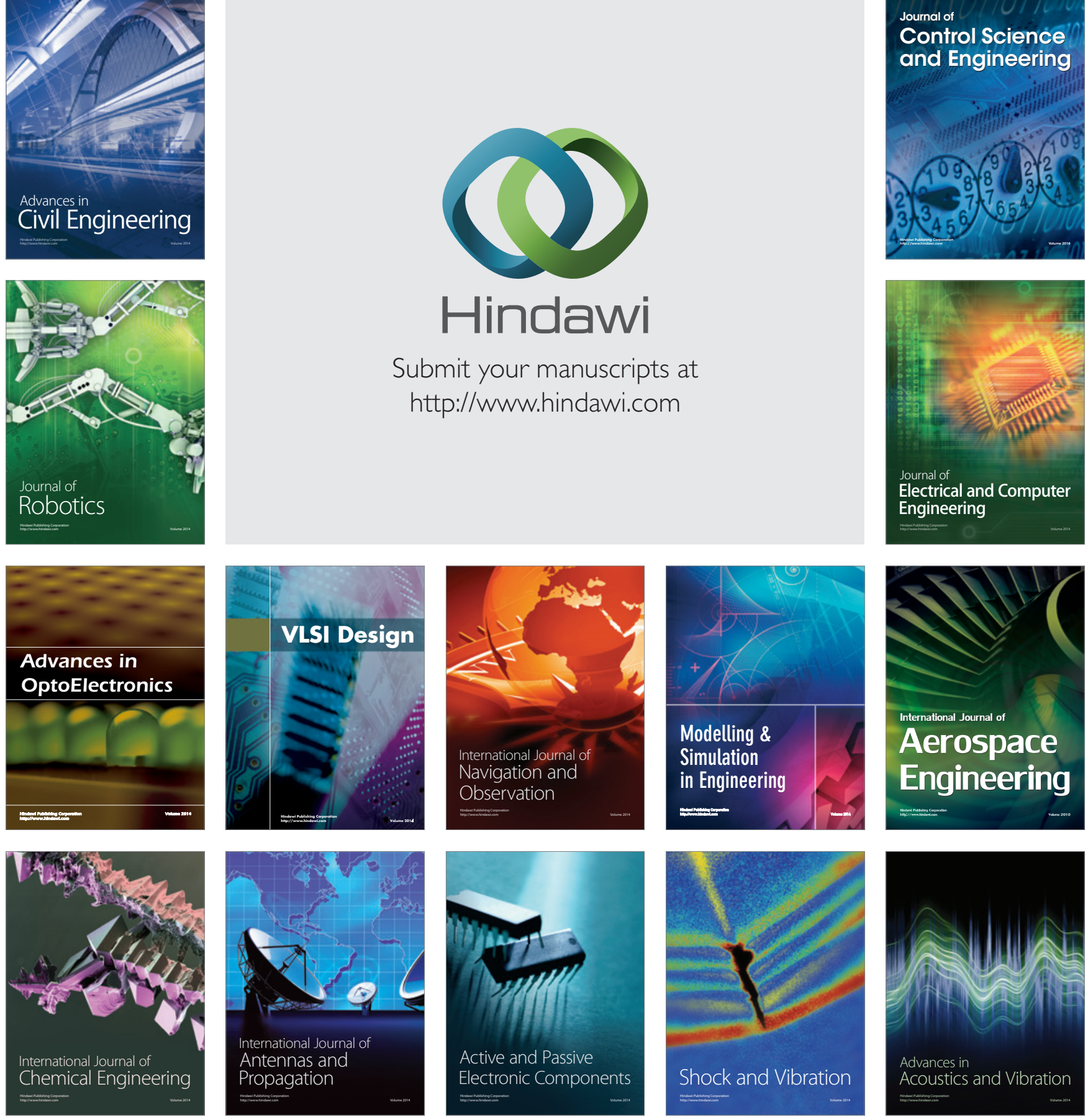